\title{
Mid-IR upconversion imaging: theory and applications
}

\author{
Pedersen, C.; Ashik, A.S.; Tidemand-Lichtenberg, Peter
}

Published in:

Proceedings of SPIE

Link to article, DOI:

$10.1117 / 12.2548283$

Publication date:

2020

Document Version

Publisher's PDF, also known as Version of record

Link back to DTU Orbit

Citation (APA):

Pedersen, C., Ashik, A. S., \& Tidemand-Lichtenberg, P. (2020). Mid-IR upconversion imaging: theory and applications. In Proceedings of SPIE (Vol. 11264). [112641A] SPIE - International Society for Optical Engineering. Proceedings of SPIE - The International Society for Optical Engineering https://doi.org/10.1117/12.2548283

\section{General rights}

Copyright and moral rights for the publications made accessible in the public portal are retained by the authors and/or other copyright owners and it is a condition of accessing publications that users recognise and abide by the legal requirements associated with these rights.

- Users may download and print one copy of any publication from the public portal for the purpose of private study or research.

- You may not further distribute the material or use it for any profit-making activity or commercial gain

- You may freely distribute the URL identifying the publication in the public portal

If you believe that this document breaches copyright please contact us providing details, and we will remove access to the work immediately and investigate your claim. 


\section{Mid-IR upconversion imaging: theory and applications}

Pedersen, C., A.S., Ashik, Tidemand-Lichtenberg, P.

C. Pedersen, Ashik A.S., P. Tidemand-Lichtenberg, "Mid-IR upconversion imaging: theory and applications," Proc. SPIE 11264, Nonlinear Frequency Generation and Conversion: Materials and Devices XIX, 112641A (2 March 2020); doi: $10.1117 / 12.2548283$

SPIE. Event: SPIE LASE, 2020, San Francisco, California, United States 


\title{
Mid-IR upconversion Imaging: Theory and applications
}

\author{
C. Pedersen*a , Ashik. A.S. ${ }^{a}$, P. Tidemand-Lichtenberg ${ }^{\mathrm{a}}$ \\ a'DTU Fotonik, Department of Photonics Engineering, Building 343, Ørsteds Plads, 2800 Lyngby, \\ Denmark
}

\begin{abstract}
Upconversion imaging using a $\chi^{(2)}$ material can conveniently be viewed as an optical filter known from Fourier optics. First part discusses the solution to Helmholtz Equation with a nonlinear source term representing the $\chi^{(2)}$ interaction process. Assuming non-depleted interaction, an explicit solution can be found using Greens Function. In the far-field the solution is found in terms of a simple 3D Fourier integral. We will analyze a 4f-setup, with the nonlinear crystal situated in the Fourier plane, for upconversion imaging. While the results resembles the linear case known from standard imaging systems, $\chi^{(2)}$ imaging has an additional phase match term, dictated by the dispersion and birefringence amongst the three interacting fields. Birefringent crystals can be implemented as the nonlinear medium to ensure phase matching, i.e. efficient conversion of the mid-IR signal to the visible. When interaction takes place in the fs regime, group velocity mismatch will be included. The main features of the theory is presented, including applications.
\end{abstract}

Keywords: Upconversion imaging, mid-IR imaging, nonlinear phase matching, sum frequency generation

\section{INTRODUCTION}

Upconversion, as a means for transferring mid-IR to the visible or near infrared wavelength region, was demonstrated already in $1968^{1}$. However, lack of good nonlinear materials and efficient pump laser designs to drive the $\chi^{(2)}$ nonlinearity prevented further exploitation, thus research in upconversion detection and imaging almost disappeared in the 1980s. Today, the advent of periodically poled crystals, cheap CCD cameras and diode pumped solid state laser systems have revived the technology. We will, in section 2, provide a short introduction to the theory of continuous wave upconversion imaging using a Fourier optics approach. The focus will be on the 4f-setup, which we will treat formally, filling in the details that were omitted in $\mathrm{ref}^{2}$. In practice, such a continuous wave $(\mathrm{CW})$ theory will be adequate for time scales down to the few ps regime. In section 3, imaging at the sub-ps time-scales will be treated. In particular, group velocity mismatch (GVM) between the interacting waves will decrease the interaction length substantially impacting the efficiency, however, at the same time increase acceptance parameters relevant for upconversion imaging. Different applications will be discussed in section 4, including both upconversion imaging and upconversion spectroscopy.

\section{CW UPCONVERSION IMAGING}

\subsection{The k-space transfer function, $h$ of $\chi^{(2)}$ upconversion imaging}

We will in this section review the central results of ref $^{2}$, as an introduction to the $4 \mathrm{f}$-setup description in section 2.2. We assume the non-depleted approximation, which is applicable when the conversion efficiency is small (e.g. $<30 \%$ ). We consider three monochromatic waves. With these restrictions, a far-field, $\mathbf{k}$-space upconversion transfer function, $h$ can be expressed as a three dimensional Fourier transform of the pump field. Writing, without loss of generality, the incident pump field as $E_{\mathrm{p}}(\mathbf{r}, t)=E_{\mathrm{p}}(\mathbf{r}) e^{-j\left(\mathbf{k}_{\mathrm{p}} \cdot \mathbf{r}-\omega_{\mathrm{p}} t\right)}$ and the infrared signal as $E_{\mathrm{IR}}(\mathbf{r}, t)=E_{\mathrm{IR}}(\mathbf{r}) e^{-j\left(\mathbf{k}_{\mathrm{IR}} \cdot \mathbf{r}-\omega_{\mathrm{IR}} t\right)}, h$ can be written as ${ }^{2}$

$$
h(\Delta \mathbf{k})=\left.\frac{2 d_{\mathrm{eff}} \omega_{\mathrm{up}}^{2}}{c^{2}} \mathcal{F}\left\{E_{\mathrm{p}}\left(\mathbf{r}^{\prime}\right)\right\}\right|_{\mathbf{f}=\frac{\Delta \mathbf{k}}{2 \pi}}
$$

where, $\Delta \mathbf{k}=\mathbf{k}_{\mathrm{up}}-\mathbf{k}_{\mathrm{IR}}-\mathbf{k}_{\mathrm{p}}$. The Fourier transform used here is defined as: $\left.\mathcal{F}\{f(\mathbf{r})\}\right|_{\mathbf{f}}=\iiint_{-\infty}^{+\infty} f(\mathbf{r}) e^{j 2 \pi \mathbf{f} \cdot \mathbf{r}} d \mathbf{r}$ 
The transfer function, $h$ describes the far-field response of the nonlinear crystal when exposed to an incident plane, monochromatic wave with $\mathbf{k}$-vector, $\mathbf{k}_{\mathrm{IR}}$. The upconverted field will have a frequency given by the relation $\omega_{\mathrm{up}}=\omega_{\mathrm{p}}+$ $\omega_{\mathrm{IR}}$. From $h$, the upconverted field for an arbitrary input field distribution can be found as a convolution process.

\subsection{Upconversion using a 4f set-up}

The k-space transfer function can be reduced to a two dimensional Fourier transform by assuming that the two input fields, $E_{\mathrm{IR}}$ an $E_{\mathrm{p}}$, do not change significantly inside the crystal as they interact (along the z-axis), or alternatively by assuming that the nonlinear volume, i.e. the $\chi^{(2)}$ crystal length, $l_{\mathrm{c}}$ is short. In that case, we can carry out the Fourier transform in $\mathrm{z}$-axis direction:

$$
h(\Delta \mathbf{k})=\left.\frac{2 d_{\mathrm{eff}} \omega_{\mathrm{up}}^{2}}{c^{2}} \mathcal{F}\left\{E_{\mathrm{p}}\left(\mathbf{r}^{\prime}\right)\right\}\right|_{\mathbf{f}=\frac{\Delta \mathbf{k}}{2 \pi}}=\left.\frac{2 d_{\mathrm{eff}} \omega_{\mathrm{up}}^{2} l_{\mathrm{c}}}{c^{2}} \mathcal{F}\left\{E_{\mathrm{p}}\left(x^{\prime}, y^{\prime}\right)\right\}\right|_{\mathbf{f}=\frac{\Delta \mathbf{k}_{T}}{2 \pi}} \operatorname{sinc}\left(\frac{\Delta k_{\mathrm{z}} l_{\mathrm{c}}}{2}\right)
$$

As seen from Eq.(1), the upconversion transfer function $h$ of the crystal can now be considered a "thin" filter as known from Fourier optics ${ }^{3}$. The z-dependency is lumped in to the well-known 'sinc' function acting on the z-axis component of $\Delta \mathbf{k}$. Since the Fourier transform in Eq.(2) is two-dimensional, we can use a 4f-setup to carry out the Fourier transform optically, see Fig.1. We have not included prefactors to highlight the simplicity of this approach.

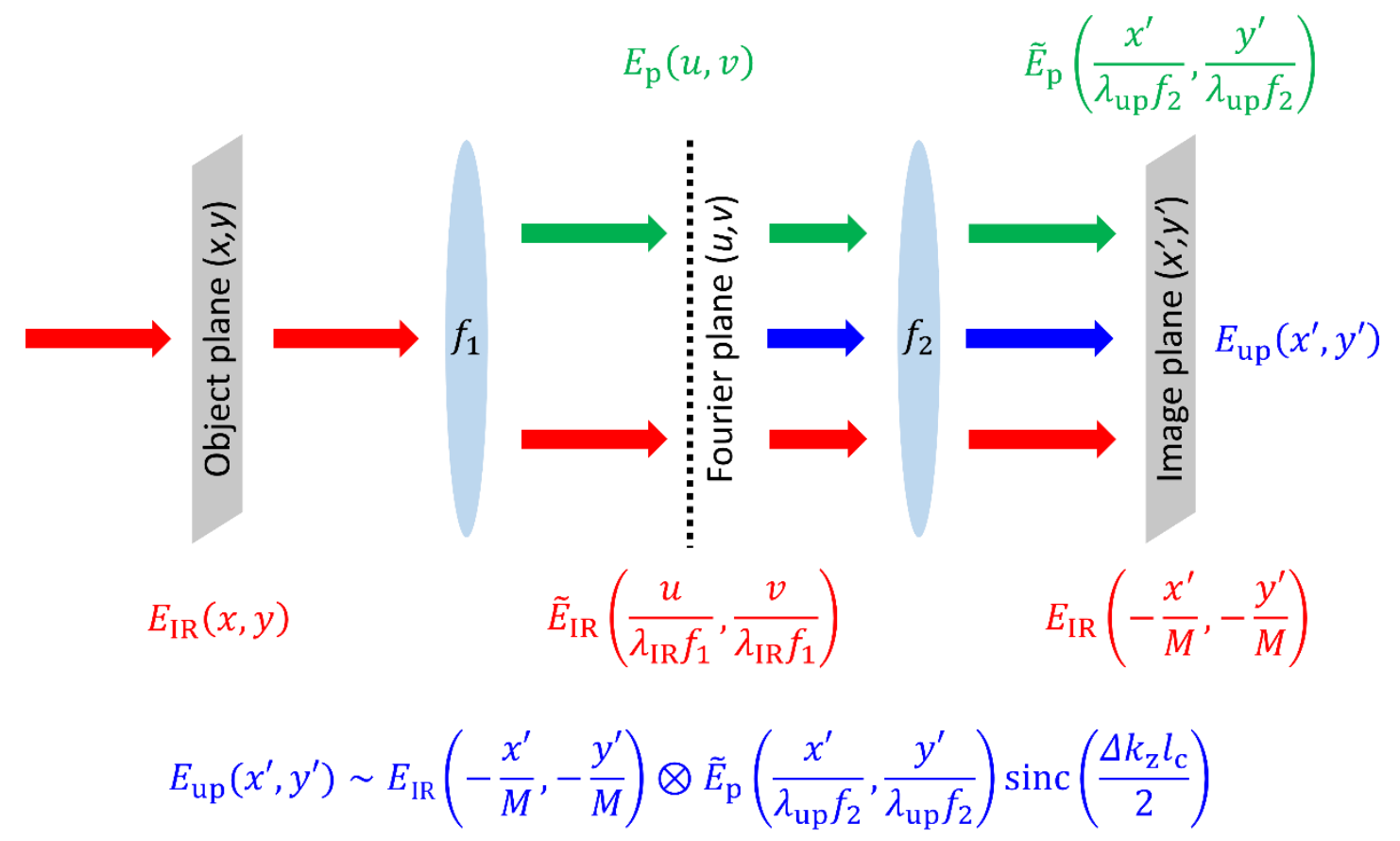

Figure 1. Fourier description of upconversion imaging using a $4 \mathrm{f}$-setup. “ ” denotes a Fourier transform, $M$ is the magnification given by $M=\left(\lambda_{\mathrm{up}} f_{2}\right) /\left(\lambda_{\mathrm{IR}} f_{1}\right)$. Arrows indicate the fields present at a position in the $4 \mathrm{f}$ system. Red, green and blue color represents the IR, pump and upconverted field respectively.

By applying the Fourier transform rules and the 2D Fourier transform properties of a lens ${ }^{3}$, the upconverted field can be found as a rescaled object field (magnification $M$ ), convolved/blurred with a point spread function, $\widetilde{E}_{\mathrm{p}}\left(\frac{x^{\prime}}{\lambda_{\mathrm{up} f_{2}}}, \frac{y^{\prime}}{\lambda_{\mathrm{up} f_{2}}}\right)$, followed by multiplication of a 'sinc' function as shown in Fig. 1. A detailed account of the interpretation can be found in refs $^{2,4}$ for the important case of a Gaussian pump beam. In physical terms, when $E_{\mathrm{p}}(u, v)$ is "constant" well beyond the transverse extent of the Fourier transformed object field, imaging will take place. 


\section{SHORT PULSE UPCONVERSION}

Short-pulsed mid-IR upconversion imaging and spectroscopy can be used for several interesting applications such as pump-probe experiments relevant for the study of relaxation dynamics of chemicals and molecules ${ }^{5}$. By short pulses, we refer to pulses having a FWHM temporal pulse width in the sub-ps regime. A myriad of short-pulsed light source exist in the mid-IR, capable of providing short pulses with high peak power, thereby eliminating the need for an intra cavity enhancement system often required for $\mathrm{CW}$ light conversion systems. High peak power facilitates single pass upconversion as described in ref $^{6}$, however, the temporal overlap between the interacting pulses, which is necessary for efficient frequency conversion, becomes progressively difficult to achieve for short pulses. One way to eschew this problem is to use the same source as pump for the upconversion process as well as for the generation of the mid-IR field ${ }^{6}$, meaning that the pump and the mid-IR pulse is synchronized passively. Such a synchronized upconversion scheme further adds to the long-term stability of the system by eliminating timing jitter and drift between the two interacting fields that could otherwise downgrade the performance of the system.

\subsection{Group Velocity Mismatch effect}

Dispersion of an optical material portray the variation in refractive index with wavelength. Dispersion leads to a difference in group velocity for pulses at different wavelengths, leading to a temporal walk-off. For long pulses ( $>$ few ps), the mismatch in group velocity is low enough so that the pulses traverse the nonlinear crystal without significant temporal walk-off. In that case, the effective interaction length is equal to the length of the crystal. This is not the case for fs pulses, where the pulses interact only in a small fraction of the crystal length before temporal walk-off separates them. The effective interaction length $\left(l_{\text {eff }}\right)$, defined as the length of the crystal over which pulses overlap, can be expressed as ${ }^{7}$

$$
l_{\text {eff }}=\frac{\tau}{\left|1 / v_{g 1}-1 / v_{g 2}\right|}
$$

where $\tau$ is the pulse duration (or more accurately the FWHM of the convolution of the two interacting pulses) and $v_{g x}$ represents the group velocity of the $x^{\text {th }}$ field. In addition to the pulse duration, the wavelength separation between the interacting fields i.e. the group velocity difference also influence the value of $l_{\text {eff }}$, as can be seen from Eq. 3 .

Acceptance parameters of an upconversion system plays a crucial role in determining the performance of the system. Assuming fixed monochromatic pump field, the spectral acceptance bandwidth $(\Delta \lambda)$ is defined as the range of incoming IR wavelengths that can be efficiently upconverted. Similarly, the angular acceptance bandwidth $(\Delta \theta)$ is defined as the range of incoming IR angles that can be efficiently upconverted. When the upconversion efficiency is low (i.e. no depletion of the pump), the upconverted intensity of a monochromatic plane IR wave (MCP), travelling collinearly with the pump beam, is proportional to $l_{\text {eff }}{ }^{2} \operatorname{sinc}^{2}\left(\left(\Delta \mathbf{k} * l_{\text {eff }}\right) / 2\right)$, where $\Delta \mathbf{k}$ represents the phase mismatch between the three interacting fields. In simple terms, $l_{\text {eff }}$ outside the 'sinc' function directly influences the upconversion efficiency whereas $l_{\text {eff }}$ inside the 'sinc' function affects the efficiency indirectly via the acceptance parameters. In order for the readers to understand more about the dynamics involved with a short pulsed upconversion system, we refer to the experimental setup shown in ref $^{6}$ where the wavelengths are centered at $0.804 \mu \mathrm{m}$ for the pump field (referred to as $\lambda_{\mathrm{p}}$ ) and $3.206 \mu \mathrm{m}$ for the IR field (referred to as $\lambda_{\mathrm{IR}}$ ). The system serves as a basis to simulate the graphs in Fig. 2. Figure 2 illustrates the influence of a GVM limited interaction length for three different pulse durations (100 fs, $1 \mathrm{ps}$, and $10 \mathrm{ps}$ ). Figure 2(a) shows the upconverted intensity, for plane wave interaction, plotted as a function of incident IR angles $\left(\theta_{\mathrm{IR}}\right)$ for a fixed IR wavelength $\left(\lambda_{\mathrm{IR}}=3.206 \mu \mathrm{m}\right)$. The angular acceptance bandwidth at different values of $\tau$, is defined as the FWHM of the respective curves in Fig. 2(a). The inset shows the corresponding change in $l_{\text {eff }}$. Note that for the considered system, $l_{\text {eff }}$ would remain constant (pink line) for $\tau$ greater than $2.15 \mathrm{ps}$ (vertical dashed line). We can clearly observe a drastic reduction of $l_{\text {eff }}$ with shorter pulse durations, increasing the angular acceptance bandwidth ( $3^{\circ}$ for $10 \mathrm{ps}$ (black curve) to $15^{\circ}$ for $100 \mathrm{fs}$ (blue curve)). Figure 2(b) shows the upconverted intensity plotted as a function of IR wavelengths for a particular incident IR angle $\left(\theta_{\mathrm{IR}}=0^{\circ}\right)$. The spectral acceptance bandwidth for different values of $\tau$, is determined by the FWHM of the respective curves in Fig. 2(b). A significant increase in the spectral acceptance bandwidth is also observed (10 nm for $10 \mathrm{ps}$ (black curve) to $200 \mathrm{~nm}$ for $100 \mathrm{fs}$ (blue curve)) as $l_{\text {eff }}$ decreases. Large angular and spectral acceptance bandwidths associated 
with such a short-pulsed upconversion system conclusively suggests an increase in the field of view (FoV). This enables wide FoV upconversion imaging or broadband spectroscopy without tuning of the phase matching condition.
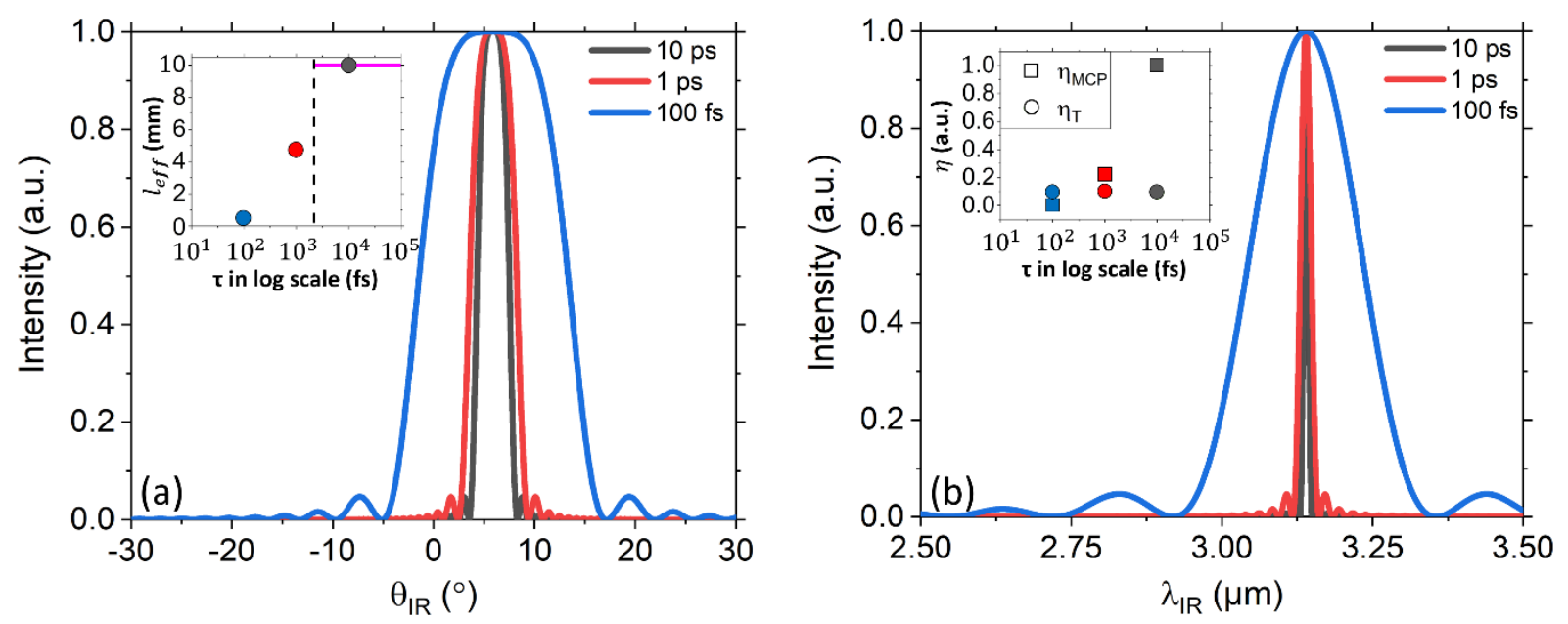

Figure 2. Illustration of the GVM limited interaction length associated with a short-pulse upconversion system. (a) shows the upconverted intensity as a function of incident IR angle for $\lambda_{\mathrm{IR}}=3.206 \mu \mathrm{m}$ and (b) shows the upconverted intensity as a function of IR wavelength for $\theta_{\mathrm{IR}}=0^{\circ}$. The insets in (a) and (b) show the influence of GVM on the effective interaction length and efficiency, respectively. $\theta_{\mathrm{IR}}$ represents external IR angles.

The upconversion efficiency $\eta_{\mathrm{T}}$ is an important parameter of an upconversion system. For the MCP case, the efficiency $\left(\eta_{\mathrm{MCP}}\right)$ is proportional to the square of $l_{\text {eff }}$ assuming phase matching. Normalizing, so that $\eta_{\mathrm{MCP}}=1$ for $l_{\mathrm{eff}}=10 \mathrm{~mm}$ (i.e. pulse width $>2.15 \mathrm{ps}$ ), the inset in Fig. 2(b) shows the reduction of $\eta_{\mathrm{MCP}}$ with $\tau$. The overall efficiency of the upconversion process $\left(\eta_{\mathrm{T}}\right)$ can be expressed as, $\eta_{\mathrm{T}} \propto \eta_{\mathrm{MCP}} \times \Delta \theta^{2} \times \Delta \lambda$. Interestingly, even though $\eta_{\mathrm{MCP}}$ decreases drastically with $\tau$ (or $l_{\text {eff }}$ ), the value of $\eta_{\mathrm{T}}$ remains almost constant for the same peak power, as observed from the inset of Fig. 2(b). This implies that the increase in the angular and spectral acceptance bandwidth compensates the decrease in efficiency due to shorter interaction length, so that the overall efficiency of the upconversion system remains almost constant. It is important to note here, that for $\eta_{\mathrm{T}}$ to remain constant, it is required that the angular and spectral distribution of the IR field should be greater than or equal to the angular and spectral acceptance bandwidth.

\subsection{Chromatic blurring effect}

Upconversion performed in the Fourier plane of a 4f-setup transforms the spatial information in the object plane into angles in the Fourier plane. As discussed in the previous section, the broad spectral acceptance bandwidth is inherent to shortpulse upconversion systems. We have experimentally observed that this leads to a non-uniform blurring artifact in the upconverted image, which is not observed in a CW system. Figure 3 illustrates the blurring effect, which we will refer to as chromatic blurring. Figure 3(a) shows the k-vector diagram for an upconversion process for two different incident plane waves (red). The phase match condition dictates that each IR angle will be upconverted to a unique upconverted angle ${ }^{6}$, shown by the thick blue lines. The grey dashed line represents the broadening of the $\mathbf{k}_{\mathrm{IR}}$ vector due to the spectral width of the IR source. In the following, we will assume that the spectral bandwidth of the source is equal to the spectral acceptance bandwidth. In that case, all the wavelength components for a particular $\theta_{\mathrm{IR}}$ will be upconverted, but into different phase matched upconverted angles, shown by the dashed blue lines. Thus, a single point in the object plane translates into a spread of angles ( $\left.\delta \theta_{\text {up }}\right)$ after upconversion. The spread in the upconverted angles translates into chromatic blurring in the image plane of the 4f-setup. Figure 3(a) further illustrates that, as the IR angle increases, the spread in the upconverted angles also increases, i.e. $\delta \theta_{\mathrm{up} 1}>\delta \theta_{\mathrm{up} 2}$ for $\theta_{\mathrm{IR} 1}>\theta_{\mathrm{IR} 2}$. For the special collinear case $\left(\theta_{\mathrm{IR}}=0^{\circ}\right)$, the upconverted field will also be collinear $\left(\theta_{\text {up }}=0^{\circ}\right)$, thus chromatic blurring will be absent $\left(\delta \theta_{\text {up }}=0^{\circ}\right)$. In $2 \mathrm{D}$ upconversion image, this impose radially increased blurring relative to the center, i.e. collinear interaction. Figure $3(\mathrm{~b})$ shows the chromatic blurring effect in an upconverted image of a USAF resolution target for the short-pulse upconversion system reported in ref ${ }^{6}$. In effect, the blurring at the center of the image is determined by the point spread function of the system 
as described in section 2, however, the broad spectral acceptance bandwidth associated with a short-pulse system leads to an additional radial increase in the blurring.
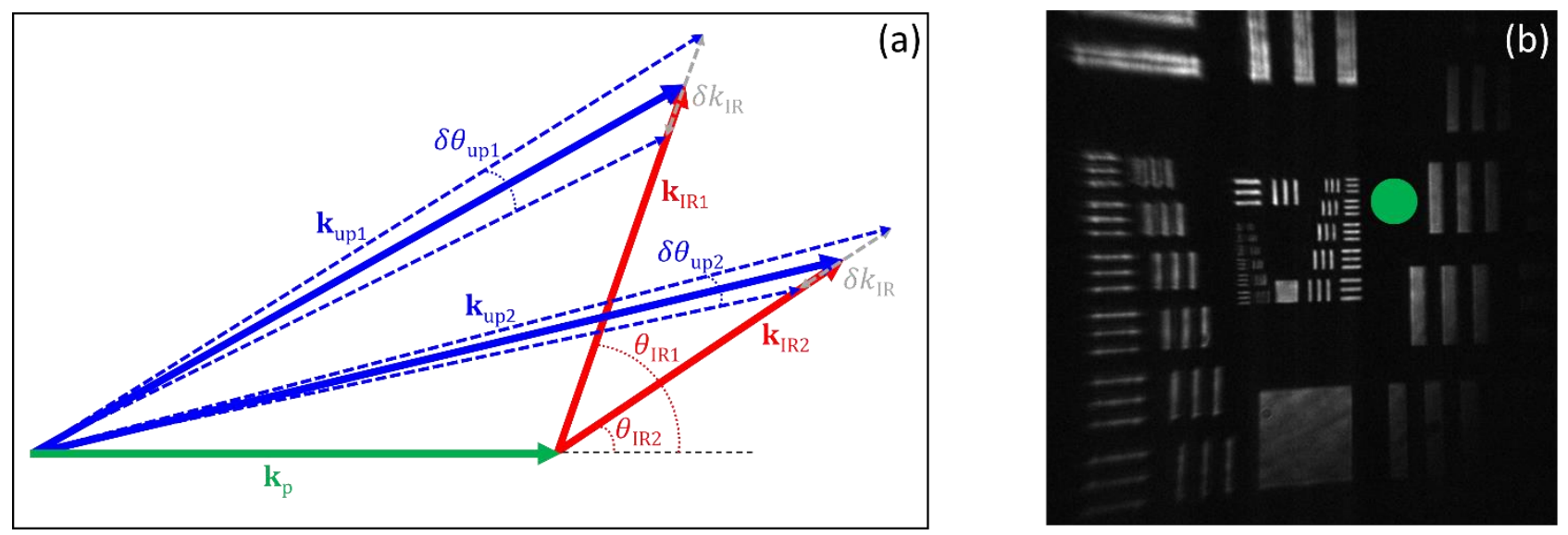

Figure 3. Illustration of chromatic blurring for short-pulsed upconversion imaging. (a) shows the wave vector diagram for the upconversion process at two different input IR angles $\left(\theta_{\mathrm{IR} 1}\right.$ and $\left.\theta_{\mathrm{IR} 2}\right) . \mathbf{k}_{\mathrm{p}}, \mathbf{k}_{\mathrm{IR}}$, and $\mathbf{k}_{\mathrm{up}}$ represents the wavevector of pump, IR and upconverted field respectively. $\delta k_{\mathrm{IR}}$ represents the spread in the IR k-vector due to the bandwidth of the source. $\delta \theta_{\text {up }}$ corresponds to the angular spread in the upconverted field. (b) shows an upconverted image recorded from the experimental setup in refThe green spot indicates the approximate location of the collinear point. Increased blurring can be observed in the radial direction from the collinear point.

\section{APPLICATIONS}

In this section, various applications of mid-IR upconversion imaging technique is discussed briefly. For a detailed discussion, readers are advised to go through each references mentioned here. Demonstration of upconversion imaging from IR to the visible happened as early as $1968^{1}$. Since then, this technology has been implemented in several applications. Upconversion imaging in the 3.2 to $3,45 \mu \mathrm{m}$ spectral region using a globar light source is reported in ref $^{8}$. Temperature tuning is implemented here to obtain a series of monochromatic images, which are then post processed to obtain an upconverted hyperspectral image. In ref $^{9}$, the object is translated to obtain hyperspectral imaging, while the phase matching condition is held constant. The speed of the imaging system is limited by the speed of mechanical translation stage. In $\mathrm{ref}^{10}$, comparison of a quantum cascade laser (narrowband) versus global (broadband) illumination in an upconversion hyperspectral imaging system in the 6 to $8 \mu \mathrm{m}$ spectral region is performed. Angular tuning is implemented to obtain the image, leading to faster acquisition.

One of the major application of upconversion detector is for detection of gases. LIDAR based system incorporating an upconversion detector has been implemented for applications like pollution monitoring ${ }^{11}$, atmospheric $\mathrm{CH}_{4}$ sensing ${ }^{12}$, and measurement of atmospheric $\mathrm{CO}_{2}$ concentration ${ }^{13}$. Another important application is in the field of histopathology. Demonstration of a video frame rate mid-IR upconversion imaging system is reported in ref $^{14}$, where label free esophageal cancer tissue diagnosis is also performed. Mid-IR upconversion based hyperspectral image (using just 62 wavelengths) of a human tissue enabled preliminary unsupervised clustering of biopsies ${ }^{14}$ and shows potential for fast computer assisted cancer biopsy screening ${ }^{15}$. Video frame rate upconversion imaging was further exploited to monitor real time butane gas leak ${ }^{14}$ indicating that this system is capable of performing real time mid-IR imaging tasks. In $\mathrm{ref}^{16}$, a real time mid-IR optical coherence tomography (OCT) system is developed integrating the conventional spectral domain OCT with a broadband upconversion detection scheme. A spectral resolution of $0.4 \mathrm{~nm}$ is observed while the depth resolution is 8.6 $\mu \mathrm{m}$. This mid-IR OCT system can be applied for non-destructive testing. Moving toward the short pulse regime, $\operatorname{ref}^{5}$ details the implementation of a short pulse upconversion system for ultrafast fluorescence spectroscopy, in particular, the study of time resolved fluorescence spectra of proteins. 


\section{CONCLUSIONS}

In this paper, we have described the concept of upconversion imaging in both the continuous wave and short pulse regime. In particular, we have illustrated a Fourier optics approach, which expedite calculations. As an important example, we treated upconversion imaging in a 4f-setup, with the nonlinear crystal in the Fourier plane. In the short pulse regime, GVM becomes increasingly important to be included in the calculation. GVM will decrease the efficiency of the system, but also broaden acceptance parameters, which in return will introduce radial, chromatic blurring in the upconverted image. Different applications of upconversion was discussed.

\section{REFERENCES}

[1] J. E. Midwinter, "Image conversion from $1.6 \mu \mathrm{m}$ to the visible in lithium niobate," Appl. Phys. Lett 12(3), 68-70 (1968)

[2] A. Barh, P. J. Rodrigo, L. Meng, C. Pedersen, and P. Tidemand-Lichtenberg, "Parametric upconversion imaging and its applications," Adv. Opt. Photon. 11(4), 952 (2019)

[3] J. W. Goodman, [Introduction to Fourier Optics], Roberts and Company Publishers (2005)

[4] C. Pedersen, E. Karamehmedović, J. S. Dam, and P. Tidemand-Lichtenberg, "Enhanced 2D-image upconversion using solid-state lasers," Opt. Express 17(23), 20885 (2009)

[5] J. Xu and J. R. Knutson, "Chapter 8 Ultrafast Fluorescence Spectroscopy via Upconversion," in [Methods in Enzymology] Elsevier, 159-183 (2008),

[6] Ashik. A. S., C. F. O’Donnell, S. Chaitanya Kumar, M. Ebrahim-Zadeh, P. Tidemand-Lichtenberg, and C. Pedersen, "Mid-infrared upconversion imaging using femtosecond pulses," Photon. Res. 7(7), 783, (2019)

[7] B. E. A. Saleh and M. C. Teich, [Fundamentals of photonics], Wiley Publishers, Chichester (2013)

[8] L. M. Kehlet, P. Tidemand-Lichtenberg, J. S. Dam, and C. Pedersen, "Infrared upconversion hyperspectral imaging," Opt. Lett. 40(6), 938 (2015)

[9] L. M. Kehlet, N. Sanders, P. Tidemand-Lichtenberg, J. S. Dam, and C. Pedersen, "Infrared hyperspectral upconversion imaging using spatial object translation," Opt. Express 23(26), 34023 (2015)

[10] S. Junaid, J. Tomko, M. P. Semtsiv, J. Kischkat, W. T. Masselink, C. Pedersen, and P. Tidemand-Lichtenberg, "Midinfrared upconversion based hyperspectral imaging," Opt. Express 26(3), 2203 (2018)

[11] H. Xia, G. Shentu, M. Shangguan, X. Xia, X. Jia, C. Wang, J. Zhang, J. S. Pelc, M. M. Fejer, Q. Zhang, X. Dou, and J-W. Pan, "Long-range micro-pulse aerosol lidar at $15 \mu \mathrm{m}$ with an upconversion single-photon detector," Opt. Lett. 40(7), 1579 (2015)

[12] L. Meng, A. Fix, M. Wirth, L. Høgstedt, P. Tidemand-Lichtenberg, C. Pedersen, and P. John Rodrigo, "Upconversion detector for range-resolved DIAL measurement of atmospheric $\mathrm{CH}_{4}$, " Opt. Express 26(4), 3850, (2018)

[13] L. Høgstedt, A. Fix, M. Wirth, C. Pedersen, and P. Tidemand-Lichtenberg, "Upconversion-based lidar measurements of atmospheric $\mathrm{CO}_{2}, "$ Opt. Express 24(5), 5152 (2016)

[14] S. Junaid, S. Chaitanya Kumar, M. Mathez, M. Hermes, N. Stone, N. Shepherd, M. Ebrahim-Zadeh, P. TidemandLichtenberg, and C. Pedersen, "Video-rate, mid-infrared hyperspectral upconversion imaging," Optica 6(6), 702 (2019)

[15] M. Hermes, R. B. Morrish, L. Huot, L. Meng, S. Junaid, J. Tomko, G. R. Lloyd, W. T. Masselink, P. TidemandLichtenberg, C. Pedersen, F. Palombo and N. Stone, "Mid-IR hyperspectral imaging for label-free histopathology and cytology," J. Opt. 20(2), 023002 (2018)

[16] N. M. Israelsen, C. R. Petersen, A. Barh, D. Jain, M. Jensen, G. Hannesschläger, P. Tidemand-Lichtenberg, C. Pedersen, A. Podoleanu and O. Bang, "Real-time high-resolution mid-infrared optical coherence tomography," Light Sci Appl 8(1), 11 (2019) 